\title{
Helical Nanofibers from Aqueous Self-Assembly of an Oligo(p-phenylene)-based
} Molecular Dumbbell

\author{
Jinyoung Bae, Jin-Ho Choi, Yong-Sik Yoo, Nam-Keun Oh, Byung-Sun Kim and Myongsoo Lee* \\ Center for Supramolecular Nano-Assembly and Department of Chemistry \\ Yonsei University, Seoul 120-749, Korea.
}

\section{Supporting Information}

Scheme 1. Synthesis of molecular dumbbell 1

\section{Experimental section}

Figure S1. MALDI-TOF mass spectrum of $\mathbf{1}$.

Figure S2. Dynamic laser light scattering (DLS) of $1(0.05 \mathrm{wt} \%)$ in aqueous solution at a scattering angle of $90^{\circ}$ (from CONTIN analysis of the autocorrelation function).

Figure S3. Angular (extrapolated to zero concentration) dependence of the DLS data for the cylindrical micelle.

Figure S4. Kratky plot ( $\square$ ) and linear fit of $\mathbf{1}$ in aqueous solution.

Figure S5. Top view (left, diameter : $8.3 \mathrm{~nm}$ ) and side view (right, pitch : $5.4 \mathrm{~nm}$ ) of a helical structure by molecular modeling. 
Scheme 1. Synthesis of Molecular Dumbbell 1

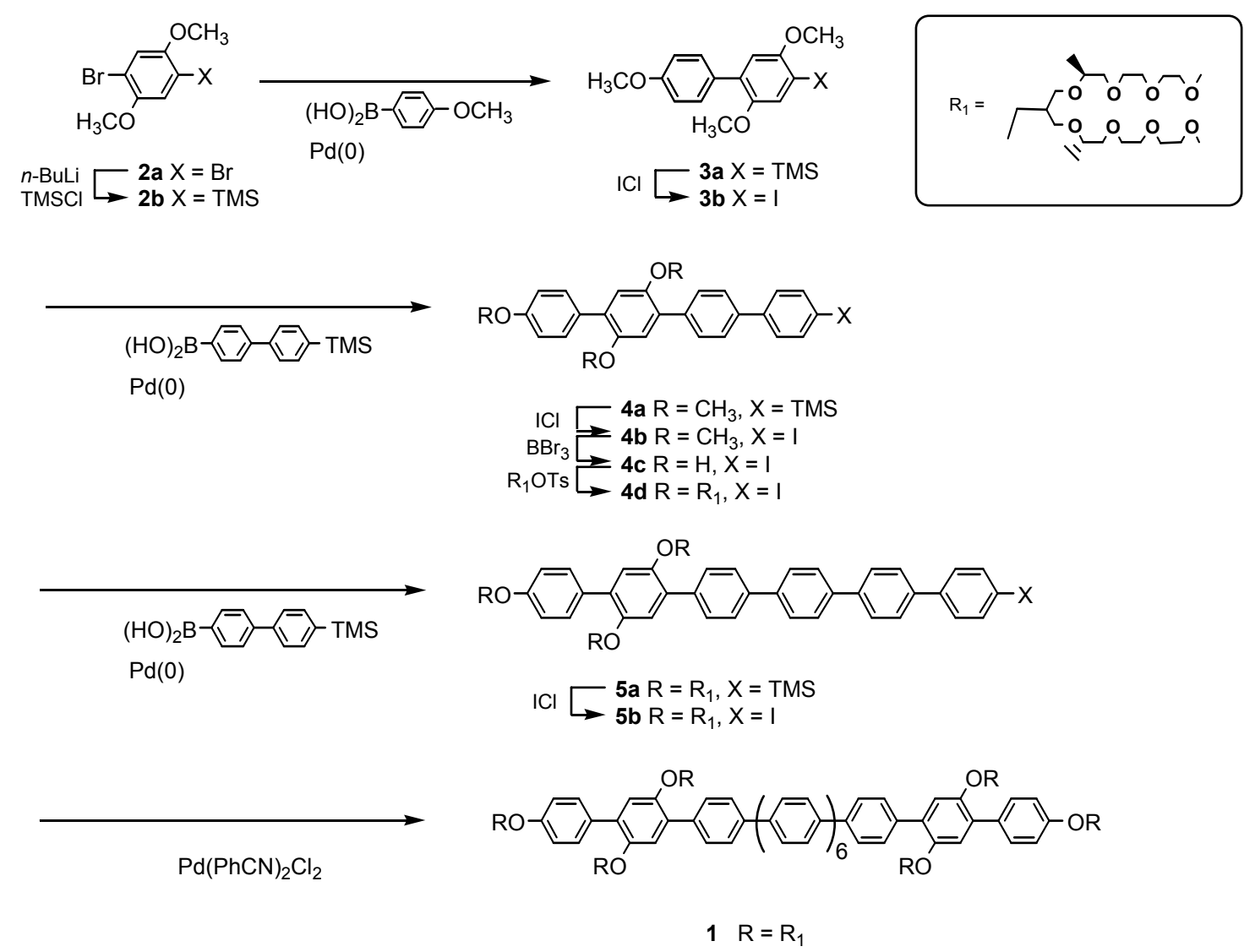




\section{Experimental section}

Materials. Chlorotrimethylsilane (98\%), tetrakis(triphenylphosphine)palladium $(0)$ (99\%), pentaerythritol (98\%), $n$-butyllithium (1.6 M solution in $n$-hexane), 4,4'dibromobiphenyl (98\%), borane-THF complex (1.0 M solution in THF), boron tribromide (1.0 M solution in dichloromethane), 18-crown-6 (99\%), potassium iodide (99\%), p-toluenesulfonyl chloride (98\%), triisopropyl borate $(98 \%)$, bisbenzonitrilepalladium(II) dichloride, tetradimethylaminoethylene (98\%), iodine monochloride (1.0 M solutuion in dichloromethane), 4-bromoanisole (99\%), (all from Aldrich) and the conventional reagents were used as received. Flash chromatography was carried out with Silica Gel 60 (230 - 400 mesh) from EM Science. Dried THF was obtained by vacuum transfer from sodium and benzophenone.

Techniques. ${ }^{1} \mathrm{H}-\mathrm{NMR}$ and ${ }^{13} \mathrm{C}$-NMR spectra were recorded from $\mathrm{CDCl}_{3}$ solutions on a Bruker AM 250 spectrometer. The purity of the products was checked by thin layer chromatography (TLC; Merck, silica gel 60). Microanalyses were performed with a Perkin-Elmer 240 elemental analyzer at Organic Chemistry Research Center, Sogang University. Molecular weight distribution $\left(M_{w} / M_{n}\right)$ were determined by gel permeation chromatography (GPC) with Waters R401 instrument equipped with Stragel HR 3, 4 and 4E columns, M7725i manual injector, column heating chamber, and 2010 Millennium data station. Measurements were made by using an UV detector, $\mathrm{CHCl}_{3}$ as solvent (1.0 mL $\cdot \min -1)$. MALDI-TOF mass spectrum was performed on a Perseptive Biosystems Voyager-DE STR using a 2,5-dihydroxy benzoic acid matrix, with dissolving 1 in $\mathrm{CH}_{2} \mathrm{Cl}_{2}$. Dynamic light scattering (DLS) measurements were performed using an ALV / CGS-3 Compact Goniometer System. Optical absorption spectra were obtained from a Shimadzu 1601 UV spectrometer. The steady-state fluorescence spectra were obtained with a Hitachi F-4500 fluorescence spectrophotometer. Circular dichroism (CD) spectra were obtained using JASCO J-810 spectropolarimeter. Band width was $2 \mathrm{~nm}$, and response time was 2 seconds. We measured repeatedly at least five times at the scan rate of $100 \mathrm{~nm} \cdot \mathrm{min}^{-1}$. The data were averaged and smoothed using JASCO software. The transmission electron microscopy (TEM) was performed at $120 \mathrm{kV}$ using JEOL 2010. For study of structure of $\mathbf{1}$ in aqueous solution, a drop of aqueous solution of molecules $1(0.05 \mathrm{wt} \%)$ was placed on a carbon-coated copper grid, and dried at room temperature. Sample was stained by depositing a drop of $2 \mathrm{wt} \%$ uranyl acetate aqueous solution onto the surface of the sample-loaded grid. Simulation was obtained with Materials Studio Modeling 3.0 (Accelrys Inc.) software. 
Synthesis. The synthesis of molecular dumbbell 1 was synthesized using a similar procedure described previously (Ref. 1, 2, and 3).

Synthesis of compound 1. Compound $5 \mathbf{b}$ ( $2.0 \mathrm{~g}, 1.07 \mathrm{mmol})$, and TDAE (0.43 g 2.15 mmol) were dissolved in DMF $(30 \mathrm{ml})$. And then $\mathrm{Pd}(\mathrm{PhCN})_{2} \mathrm{Cl}_{2}(0.02 \mathrm{~g}, 0.05 \mathrm{mmol})$ was added. The mixture was heated at $50{ }^{\circ} \mathrm{C}$ for $4 \mathrm{hrs}$ with stirring under nitrogen. Cooled to room temperature, the DMF solvent were removed under vacuum distillation, and the crude products was purified by column chromatography (silica gel, ethyl acetate followed by THF) and precipitated by $\mathrm{CH}_{2} \mathrm{Cl}_{2}$ and $n$-hexane to yield $1.4 \mathrm{~g} \mathrm{(77 \% )} \mathrm{of} \mathrm{a}$ yellow liquid ; GPC $\mathrm{M}_{\mathrm{w}} / \mathrm{M}_{\mathrm{n}}=1.03,{ }^{1} \mathrm{H}-\mathrm{NMR}\left(250 \mathrm{MHz}, \mathrm{CDCl}_{3}, \delta\right.$, ppm) 0.99-1.14 (m, $\left.36 \mathrm{H}, \mathrm{CHCH}_{3}\right), 2.13-2.25\left(\mathrm{~m}, 6 \mathrm{H}, \mathrm{C}\left(\mathrm{CH}_{2}\right)_{3} \mathrm{H}\right), 3.31-3.41\left(\mathrm{~m}, 12 \mathrm{H}, \mathrm{OCH}_{3}\right), 3.49-3.65$ (m, $\left.116 \mathrm{H}, \mathrm{OCH}_{2}\right), 4.02\left(\mathrm{~s}, 12 \mathrm{H}, \mathrm{CH}_{2} \mathrm{OPh}\right), 6.83-6.98\left(\mathrm{~m}, 8 \mathrm{Ar}-\mathrm{H}, o\right.$ to $\left.\mathrm{OCH}_{2}\right), 7.46(\mathrm{~d}, 4 \mathrm{Ar}-\mathrm{H}$, $m$ to $\left.\mathrm{OCH}_{2}, J=8.6 \mathrm{~Hz}\right), 7.63-7.79(\mathrm{~m}, 32 \mathrm{Ar}-\mathrm{H}){ }^{13} \mathrm{C}-\mathrm{NMR}\left(250 \mathrm{MHz}, \mathrm{CDCl}_{3}, \mathrm{ppm}\right): \delta$ $=158.18,149.99,139.46,138.79,137.55,130.45,130.39,129.99,129.35,127.33$, 126.29, 115.26, 113.81, 74.98, 74.83, 74.69, 71.83, 71.79, 70.67, 70.61, 70.54, 70.49, 70.46, 67.07, 66.11, 58.94, 40.97, 40.95, 17.08, 16.99, 16.95, 16.90 Anal. Calcd for $\mathrm{C}_{132} \mathrm{H}_{186} \mathrm{O}_{36}$ : C, 67.50; H, 7.98 Found C, 67.46 ; H, 7.95.

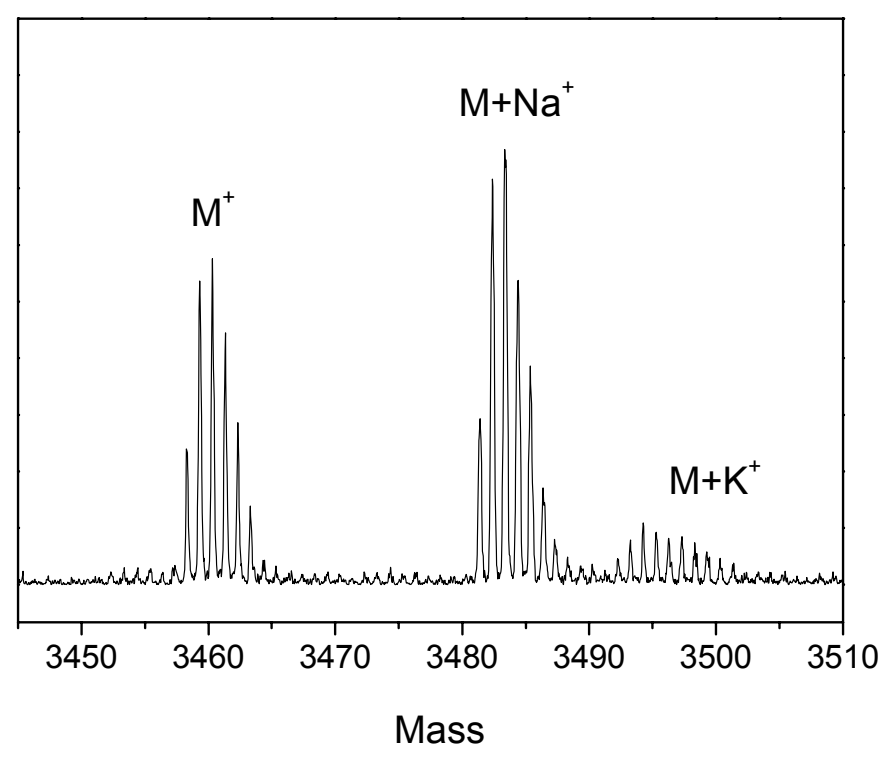

Figure S1. MALDI-TOF mass spectrum of $\mathbf{1}$. 


\section{DLS Experiment}

Dynamic light scattering (DLS) experiments were performed with 1 in aqueous solution in order to investigate the aggregation behavior. The CONTIN analysis of the autocorrelation function shows a broad peak corresponding to an average hydrodynamic radius of approximately $270 \mathrm{~nm}$. The angular dependence of the apparent diffusion coefficient $\left(D_{a p p}\right)$ was measured because the slope is related to the shape of the diffusing species. The slope was observed to be 0.02 , consistent with the value predicted for cylindrical micelles (0.03) (Ref. 4). The formation of cylindrical micelles was further confirmed by the Kratky plot that shows a linear angular dependence over the scattering light intensity of the aggregates (Ref. 5).

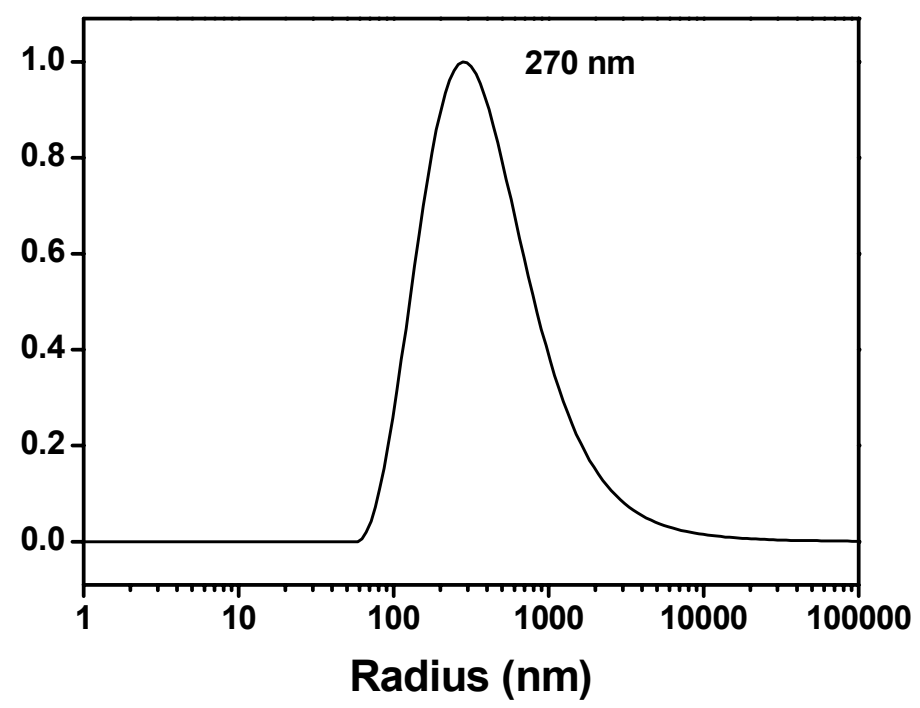

Figure S2. Dynamic laser light (DLS) scattering of 1 (0.05 wt \%) in aqueous solution at a scattering angle of $90^{\circ}$ (from CONTIN analysis of the autocorrelation function). 


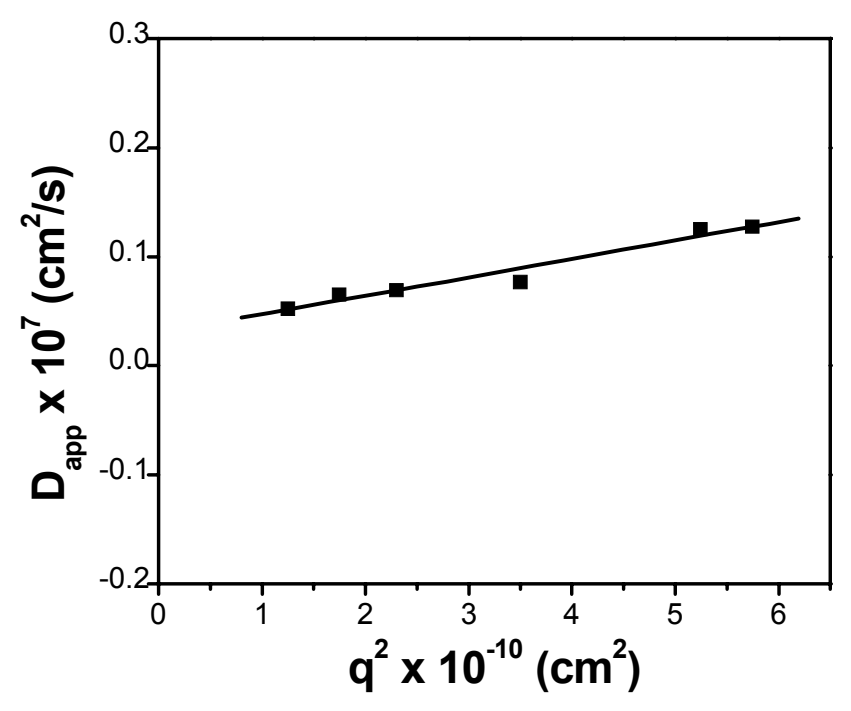

Figure S3. Angular (extrapolated to zero concentration) dependence of the DLS data for the cylindrical micelle.

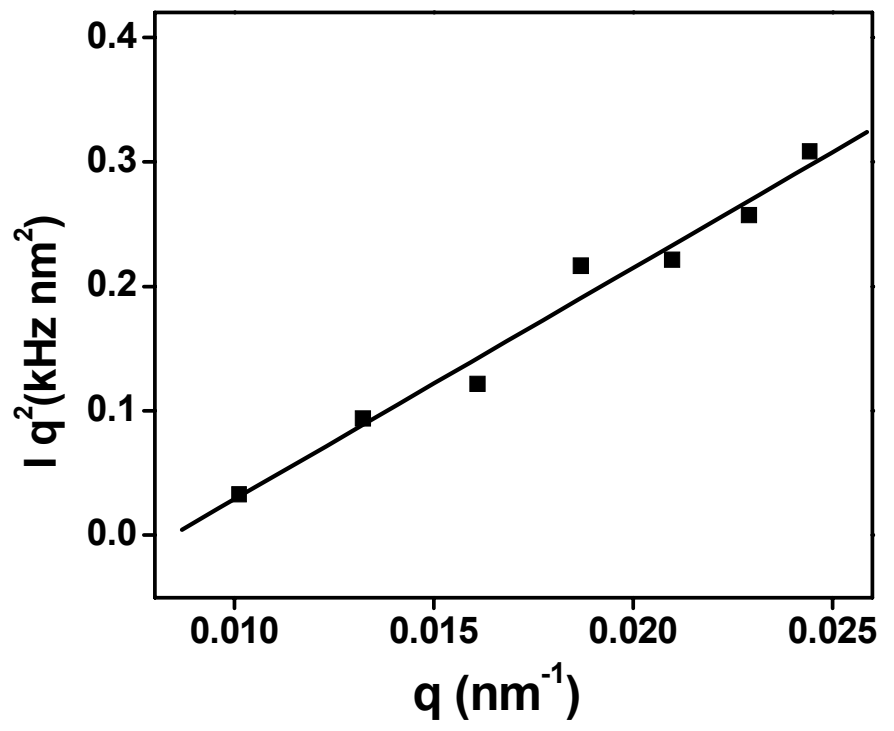

Figure S4. Kratky plot ( $\square$ ) and linear fit of $\mathbf{1}$ in aqueous solution. 


\section{Simulation}

Simulation was obtained according to the following procedure. First, a pitch of helix consisting of 12 molecules 1 was built using the Visualizer. The potential energy of helix was then minimized until the root-mean square derivative was $1.0\left(\mathrm{kcal} \cdot \mathrm{mol}^{-1}\right)$. $\AA^{-1}$ or less. The constant temperature, constant density condition (NVT ensemble) was performed on the minimized structure. The structural energy minimization of a pitch of helix was carried out for $100.0 \mathrm{ps}$ at $298 \mathrm{~K}$, a time step of $0.001 \mathrm{ps}$ and number of steps of 100000 . The interatomic interactions were modeled with the COMPASS force field. (Ref.6)
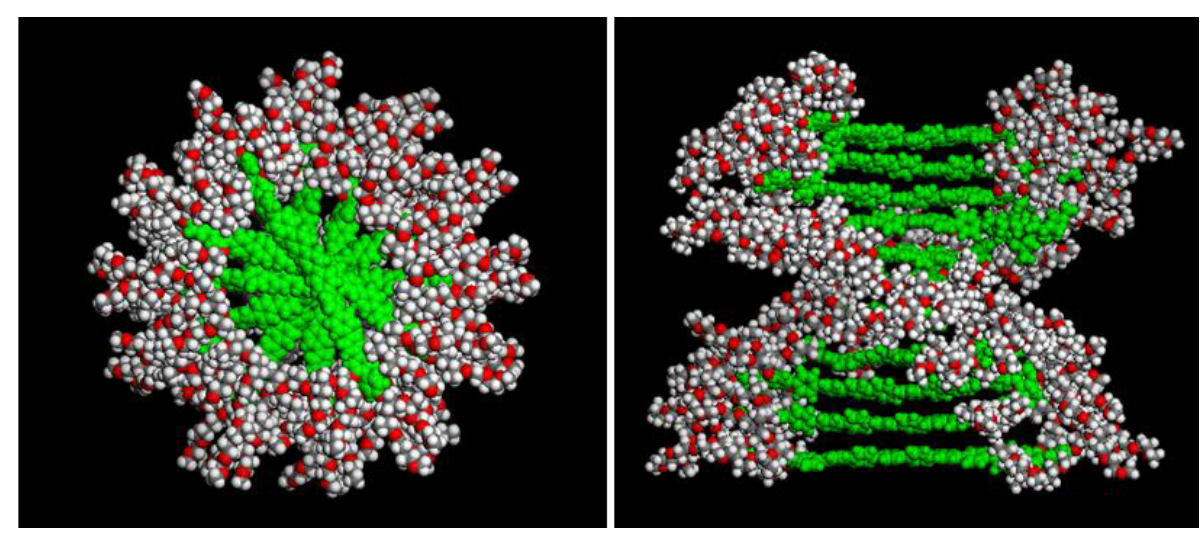

Figure S5. Top view (left, diameter : $8.3 \mathrm{~nm}$ ) and side view (right, pitch : $5.4 \mathrm{~nm}$ ) of a helical structure by molecular modeling.

\section{Supporting references}

Ref.1. Vahlenkamp, T.; Wengner, G. Macromol. Chem. Phys. 1994, 195, 1933-1952

Ref.2. Kim, H.-J.; Zin, W.-C.; Lee, M. J. Am. Chem. Soc. 2004, 126, 7009-7014

Ref.3. Yoo, Y.-S.; Choi, J.-H.; Song, J.-H.; Oh, N.-K.; Zin, W.-C.; Park, S.; Chang, T.; Lee, M. J. Am. Chem. Soc. 2004, 126, 6294-6300

Ref.4. Gohy, J.-F.; Lohmeijer, B. G. G.; Alexeev, A.; Wang, X.-S.; Manners, I.; Winnik, M. A.; Schubert, U. S. Chem. Eur. J. 2004, 10, 4315-4323

Ref.5. Bockstaller, M.; Köhler, W.; Wegner, G.; Vlassopoulos, D.; Fytas, G. Macromolecules 2000, 33, 3951-3953

Ref.6. H. Sun, J. Phys. Chem. B 1998, 102, 7338-7364 